\title{
A rare cause of renal colic pain: Chilaiditi syndrome
}

\author{
Murat Tuncer, Cahit Sahin, Ozgur Yazici, Alper Kafkaslı, Kemal Sarica \\ Dr. Lutfi Kirdar Training and Research Hospital Urology Clinic, Istanbul, Turkey.
}

\begin{abstract}
Summary Chilaiditi syndrome, first described in 1910 by the radiologist Chilaiditi from Vienna, is the interposition of right colon between liver and right hemi diaphragm. It occurs most often in males and its incidence increases with age. It is often detected incidentally during radiological examination. It's rarely symptomatic; symptoms can differ from mild abdominal pain to severe acute intestinal obstruction. Our case applied to emergency service with right flank pain.

There was no calculus or dilatation in the urinary system at non-contrast abdominopelvic computerized tomography. Ascending colon was interposed between liver and diaphragm so that the patient was diagnosed as Chiliaditi syndrome. The patient was treated conservatively and discharged with dietary suggestions by the gastroenterology consultant. The conclusion of this report is that the Chilaiditi syndrome must be considered in differential diagnosis for patients presenting with urinary colic pain symptoms with no urinary pathology on radiologic imaging.
\end{abstract}

KEY WORDS: Chilaiditi Syndrome; Renal colic; Hepatodiaphragmatic interposition.

Submitted 3 February 2014; Accepted 30 June 2014

\section{Case report}

We present a patient with Chilaiditi syndrome referred to emergency department for severe right renal colic pain, who was diagnosed with the help of radiological examinations and treated conservatively.

Case report details in Supplementary Materials posted on www.aiua.it

\section{Discussion}

As in the majority of the cases of asymptomatic anatomical abnormalities, Chilaiditi's sign is a characteristic radiological finding of hepatodiaphragmatic interposition of bowel segment. As Chiladiti syndrome has no specific clinical finding(s) which will let the clinician to consider the pathology at once and make the diagnosis, this pathology is usually incidentally diagnosed during a routine chest and/or abdominal plain film (4) whereas CT and/or ultrasonography examination have been reported to be necessary for the differential diagnosis. Although the majority of the cases are clinically symptom free, in case of associated symptoms (abdominal pain, nausea, vomiting, distension, anorexia, constipation, respiratory distress and chest pain $(2,6,7)$ it is called Chilaiditi syndrome (8). The pathology is extremely rare and up to now approximately a total of 160 cases have been reported in the literature (9). In our present case radiological images were not obtained during an asymptomatic period but CT evaluation done during symptomatic period confirmed the diagnosis.

Hepatodiaphragmatic interposition of right colon is the most common radiological sign of Chiliaditi syndrome. Although an anterior interposition is the most common radiologic finding; posterior interposition is also possible in a certain percent of the cases (7). On the other hand, ileal or gastric form of interpositions have also been described in the literature (7). This condition may be permanent or temporary (10).

Although the precise underlying causes of this pathologic interposition are still to be clarified, some liver (small or ptotic liver, cirrhosis, abnormal or deficient falciform ligament), diaphragm (diaphragmatic muscle degeneration, phrenic nerve palsy, and intrathoracic pressure increase due to tuberculosis or emphysema) and lastly colon related factors (abnormal dilatation of colon, abnormal or deficient suspensory ligament and congenital malposition or malrotation of colon, chronic constipation, aerophagia) could be responsible for this anatomical abnormal location of the colon $(2,11)$.

The differential diagnoses of Chilaiditi syndrome can also include bowel obstruction, volvulus, intussusception, ischemic bowel, or inflammatory conditions (eg, appendicitis or diverticulitis) and diaphragmatic hernia (2) pneumoperitoneum and subphrenic abcesses (12). In our case, at physical examination, lung auscultation was normal and there was no rebound or defence during abdominal palpation. Furthermore there were no signs of infection like fever and leucocytosis.

In the light of the present clinical signs, symptoms and laboratory findings along with the normal anatomy of the gallbladder on CT (which may cause right upper quadrant abdominal pain), and absence of other characteristic radiologic signs which may be attributed to other well known pathologies (volvulus, intussusception, ischemic bowel or inflammatory conditions such as appendicitis or diverticulitis, etc.) and should be considered in differential diagnosis we took in consideration this syndrome. Colonic

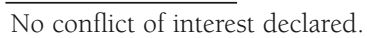


interposition between liver and diaphragm and the presence of aforementioned symptoms, made us to diagnose the case as a Chilaiditi syndrome.

This syndrome is generally asymptomatic however patients can refer with symptoms of abdominal pain, nausea, vomiting, distension, anorexia, constipation, respiratory distress, cardiac arrhythmia (12). Occasionally, it may be associated with some severe complications such as internal hernias, colonic volvulus and acute intestinal obstruction (7).

Treatment of Chilaiditi syndrome is generally conservative. This approach requires bed rest, nasogastric and/or rectal decompression, high fiber diet, fluid supplementation and stool softeners in symptomatic cases (7). Although conservative management is successful to relieve the existing symptoms in the majority of the cases, surgical treatment (such as subtotal colectomy, peritoneal fixation of colon, and hepatopexy) may be necessary in cases with persistent pain, refractory ileus, colonic volvulus or bowel ischemia $(10,13)$. Conservative management was successful in our case and the clinical course was uneventful without any serious complication.

Chilaiditi syndrome generally presents with gastrointestinal, respiratory and cardiac symptoms. However, patients can rarely refer with symptoms mimicking renal colic pain as shown in our present case. To our knowledge there is only one case with this syndrome reported in the literature referring with renal colic symptoms (14) although another case has been reported to have urological problems such as complaints of prostatism and right renal stone (15).

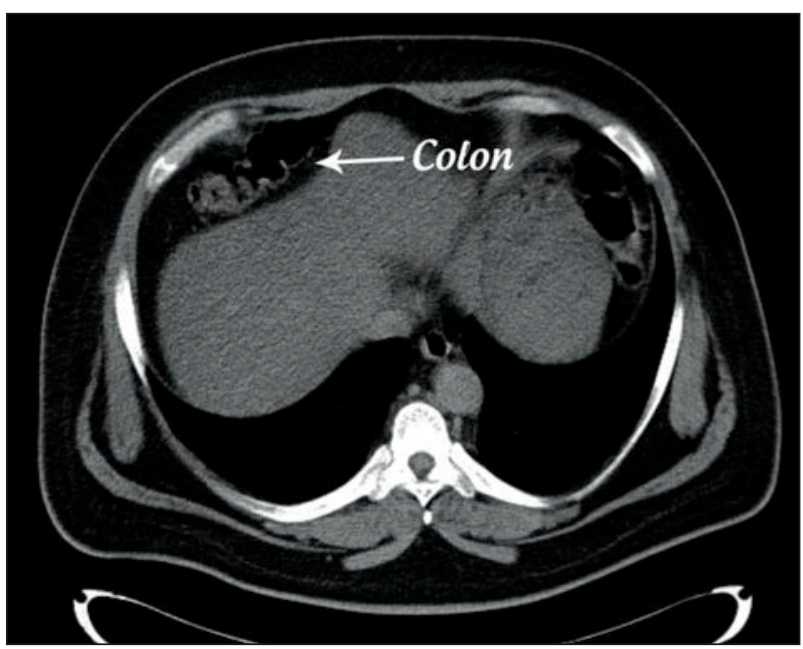

Interposition of colon between diaphragm and liver: the Chilaiditi sign.

\section{REFERENCES}

1. Chilaiditi D. On the question of the hepatoptosis ptosis and generally in the exclusion of three cases of temporary partial liver displacement. Progr Field Roentgenst. 1910; 11:173-208.

2. Moaven O, Hodin RA. Chilaiditi syndrome: a rare entity with important differential diagnoses. Gastroenterol Hepatol. 2012; 4: $276-8$.

3. McNamara RF, Cusack S, Hallihan P. Chilaiditi's syndrome. West J Emerg Med. 2009; 10:250.

4. Chen SY, Liu CT, Tsai YC, et al. Sigmoid volvulus associated Chilaiditi's syndrome. Rev Esp Enferm Dig. 2007; 99:482-3.

5. Dogu F, Reisli I, Ikinciogullari A, et al. Unusual cause of respiratory distress: Chilaiditi syndrome. Pediatrics International. 2004; 46:188-190.

6. Angulo Cuesta J, González Zorraquino A, Unda Urzaiz M, Flores Corral N. Chilaiditi syndrome in the differential diagnosis of renal colic. Arch Esp Urol. 1991; 44:300-1.

7. Qubenarssa A, Perrault LP, Ridoux G, et al. Hepatodiaphragmatic interposition of the colon: an unusual case of combined anterior and posterior types treated with an original operative technique. Dis Colon Rectum. 1999; 42:278-80.

8. Sorrentino D, Bazzocchi M, Badano L, et al. Heart-touching Chilaiditi's syndrome. World J Gastroenterol. 2005; 11:4607-9.

9. Yagnik VD. Chilaiditi syndrome with carcinoma rectum. Saudi J Gastroenterol. 2011; 17:85-6.

10. Haddad CJ, Lacle J. Chilaiditi's syndrome: a diagnostic challange. Postgrad Med. 1991; 89:249-52.

11. White JJ, Chavez EP, Macon SJ. Internal hernia of the transverse colon Chilaiditi Syndrome in a child. J Pediatr Surg. 2002; 37:802-4.

12. Dogu F, Reisli I, Ikinciogullari A, et al. Unusual cause of respiratory distress: Chilaiditi syndrome. Pediatr Int. 2004; 46:188-90.

13. Hsu HL, Liu KL. Hepatodiphragmatic interposition of the colon.CMAJ. 2011; 183:132.

14. Alva S, Shetty-Alva N, Longo WE. Image of the month. Chilaiditi sign or syndrome.Arch Surg 2008; 143:93-4.

15. Özer C, Zenger S. Chilaiditi syndrome in a patient with urological problems: Incidental diagnosis on computed tomography. Can Urol Assoc J. 2012; 6:75-6.

\section{Correspondence}

Murat Tuncer, MD (Corresponding Author)

murattuncer77@hotmail.com.

Cahit Sahin, MD

cahitsahin129@hotmail.com

Ozgur Yazici, MD

md.ozguryazici@yahoo.com.tr

Alper Kafkasl, MD

alpkafkasli@hotmail.com

Kemal Sanca, MD Professor

saricakemal@gmail.com

Altunizade mah.Atif bey sok.Gokdeniz sitesi E blok D:20 Kosuyolu, Istanbul, Turkey 